\title{
Examination of a Special Function Defined by an Integral
}

\author{
H. Volkan Ersoy \\ Department of Mechanical Engineering, Yildiz Technical University, Istanbul, Turkey \\ Email: hversoy@yildiz.edu.tr
}

Received September 14, 2011; revised October 3, 2011; accepted October 15, 2011

\begin{abstract}
The aim of this short note is to examine the properties of a special function defined by an integral which was appeared in a paper by Ersoy. It is revealed that the function $A_{r}(m, n)$ for $m=1$ is expressed in terms of the gamma function and it varies linearly with $m$ for $n \rightarrow \infty$. Its appropriate graphs are plotted and its pertinent values are tabulated.
\end{abstract}

Keywords: Special Function; Complementary Error Function; Gamma Function

\section{Introduction}

Ersoy [1] obtained a series solution which is rapidly convergent at small times while he investigated an expression for velocity field of an unsteady flow between eccentric rotating disks. In this short note, the properties of the special function used in the series solution are presented.

\section{Definition of the Function $A_{r}(m, n)$}

The function $A_{r}(m, n)$ is defined as follows:

$$
\begin{gathered}
A_{r}(m, n)=\sum_{p=0}^{\infty}\left[\mathrm{i}^{r} \operatorname{erfc} \frac{1+2 p-m}{2 n}-\mathrm{i}^{r} \operatorname{erfc} \frac{1+2 p+m}{2 n}\right] \\
(r=0,1,2, \cdots,-1 \leq m \leq 1,0<n<\infty)
\end{gathered}
$$

where

$$
\begin{aligned}
& \mathrm{i}^{n} \operatorname{erfc} x=\int_{x}^{\infty} \mathrm{i}^{n-1} \operatorname{erfc} \phi \mathrm{d} \phi \quad(n=0,1,2, \cdots), \\
& \mathrm{i}^{-1} \operatorname{erfc} x=\frac{2}{\sqrt{\pi}} e^{-x^{2}}, \\
& \mathrm{i}^{0} \operatorname{erfc} x=\operatorname{erfc} x
\end{aligned}
$$

$\operatorname{erfc} x$ and $i^{n} \operatorname{erfc} x$ denote the complementary error function and the repeated integrals of the complementary error function, respectively [2].

\section{Main Results}

In order to acquire the properties of the function $A_{r}(m, n)$, computer-assisted research is done. Furthermore, the illustrative graphs are shown in Figures 1-6 and the elucidative values are provided in Tables 1-7. The results are noted as follows:
- The function $A_{r}(m, n)$ that is a continuous function is an odd function of $m$, i.e.,

$$
A_{r}(m, n)=-A_{r}(-m, n) \text {. }
$$

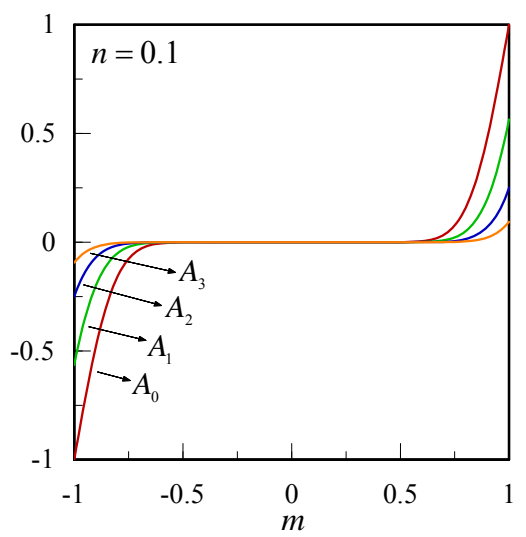

Figure 1. Variation of $A_{r}(m, n)$ with $m(n=0.1, r=0,1,2$, 3).

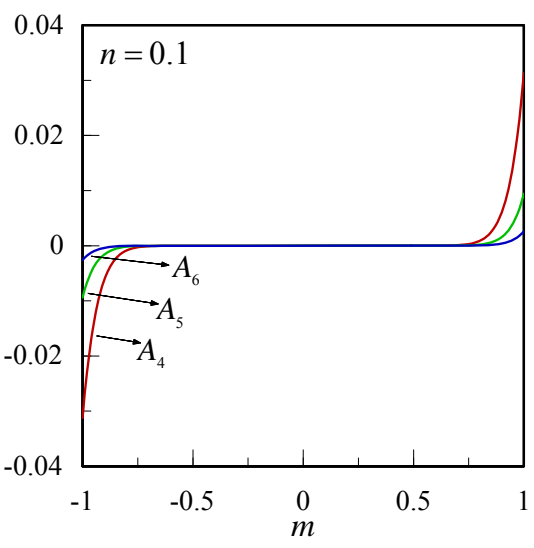

Figure 2. Variation of $A_{r}(m, n)$ with $m(n=0.1, r=4,5,6)$. 


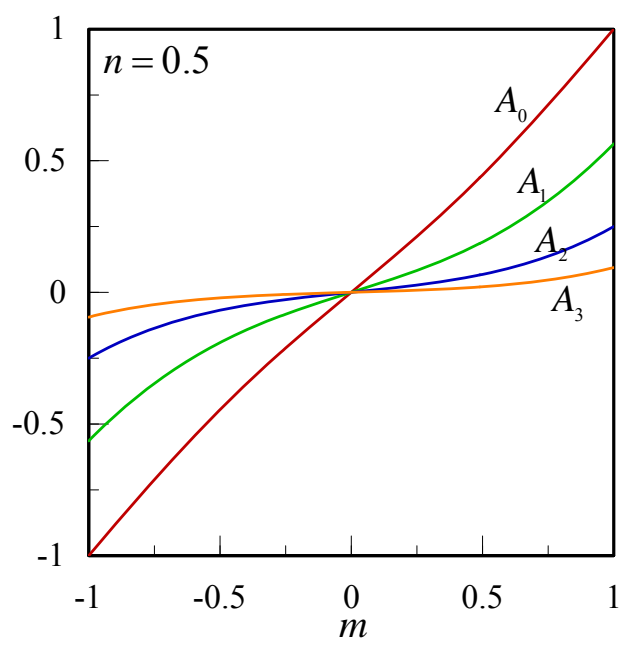

Figure 3. Variation of $A_{r}(m, n)$ with $m(n=0.5, r=0,1,2$, 3).

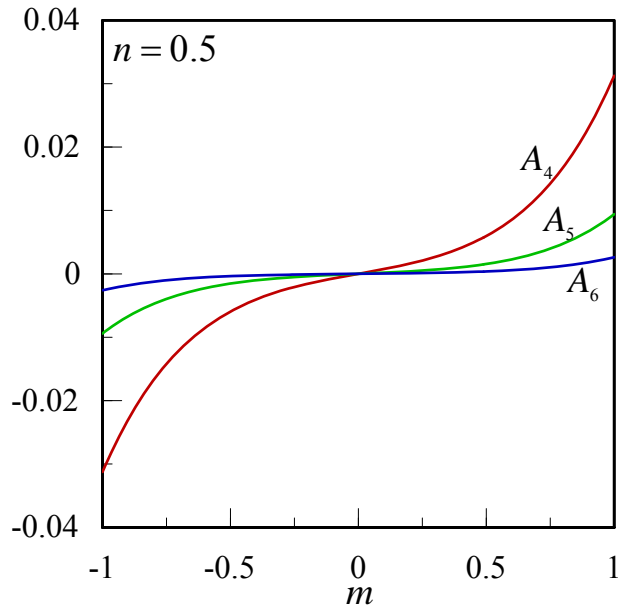

Figure 4. Variation of $A_{r}(m, n)$ with $m(n=0.5, r=4,5,6)$.

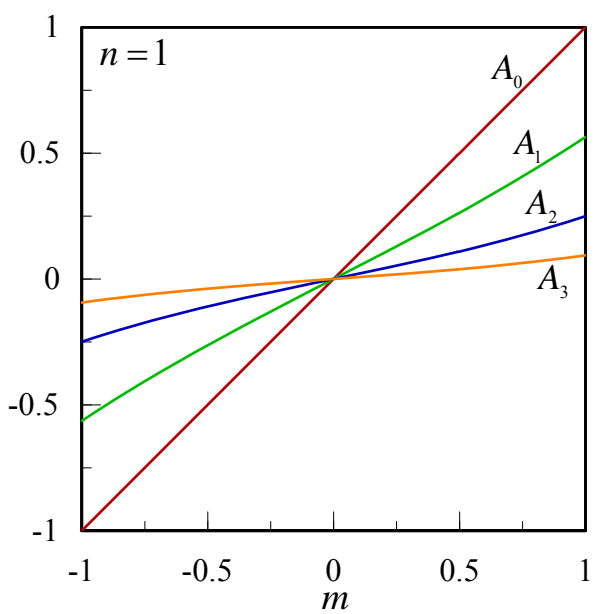

Figure 5. Variation of $A_{r}(m, n)$ with $m(n=1, r=0,1,2$, 3).

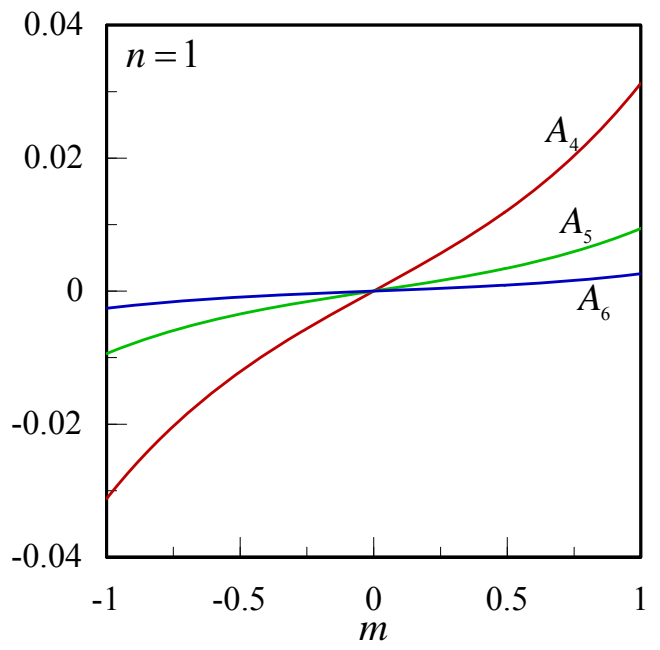

Figure 6. Variation of $A_{r}(m, n)$ with $m(n=1, r=4,5,6)$.

Table 1. Some pertinent values of $A_{0}(m, n)$.

\begin{tabular}{ccc}
\hline$A_{0}(m, n)$ & $m=0.2$ & $m=0.4$ \\
\hline$n=0.1$ & 0.000000015417 & 0.000022090497 \\
$n=0.2$ & 0.004655644484 & 0.033894110426 \\
$n=0.5$ & 0.168282000967 & 0.348663541415 \\
$n=1.0$ & 0.199980645405 & 0.399968683608 \\
$n \rightarrow \infty$ & 0.2 & 0.4 \\
\hline$A_{0}(m, n)$ & $m=0.6$ & $m=0.8$ \\
\hline$n=0.1$ & 0.004677734981 & 0.157299207050 \\
$n=0.2$ & 0.157299191633 & 0.479500121990 \\
$n=0.5$ & 0.548644186821 & 0.768250684575 \\
$n=1.0$ & 0.599968683608 & 0.799980645405 \\
$n \rightarrow \infty$ & 0.6 & 0.8 \\
\hline$A_{0}(m, n)$ & $m=1.0$ & \\
$n=0.1$ & 1.0 & \\
$n=0.2$ & 1.0 & \\
$n=0.5$ & 1.0 & \\
$n=1.0$ & 1.0 & \\
$n \rightarrow \infty$ & & \\
\hline
\end{tabular}

It becomes zero for $m=0$, i.e.,

$$
A_{r}(0, n)=0 \text {. }
$$

- The function $A_{r}(m, n)$ has the following relation for fixed $m$ and $n$ :

$$
A_{0}(m, n)>A_{1}(m, n)>A_{2}(m, n)>\cdots
$$

- The function $A_{r}(m, n)$ increases with $m$ for fixed 
Table 2. Some pertinent values of $A_{1}(m, n)$.

\begin{tabular}{ccc}
\hline$A_{1}(m, n)$ & $m=0.2$ & $m=0.4$ \\
\hline$n=0.1$ & 0.000000001822 & 0.000003355035 \\
$n=0.2$ & 0.000974667680 & 0.008622765456 \\
$n=0.5$ & 0.065135918164 & 0.143305476726 \\
$n=1.0$ & 0.103175516893 & 0.208828584303 \\
$n=2.0$ & 0.110547450789 & 0.221670644993 \\
$n \rightarrow \infty$ & 0.112837916710 & 0.225675833419 \\
\hline$A_{1}(m, n)$ & $m=0.6$ & $m=0.8$ \\
\hline$n=0.1$ & 0.000978022715 & 0.050254541660 \\
$n=0.2$ & 0.050254539838 & 0.199641228353 \\
$n=0.5$ & 0.246480993620 & 0.384511953256 \\
$n=1.0$ & 0.319376035092 & 0.437117847144 \\
$n=2.0$ & 0.333942330251 & 0.447929309378 \\
$n \rightarrow \infty$ & 0.338513750129 & 0.451351666838 \\
\hline$A_{1}(m, n)$ & $m=1.0$ & \\
\hline$n=0.1$ & 0.564189583548 & \\
$n=0.2$ & 0.564189583548 & \\
$n=0.5$ & 0.564189583548 & \\
$n=1.0$ & 0.564189583548 & \\
$n=2.0$ & 0.564189583548 & \\
$n \rightarrow \infty$ & 0.564189583548 & \\
\hline & &
\end{tabular}

Table 3. Some pertinent values of $A_{2}(m, n)$.

\begin{tabular}{ccc}
\hline$A_{2}(m, n)$ & $m=0.2$ & $m=0.4$ \\
\hline$n=0.1$ & 0.000000000210 & 0.000000490072 \\
$n=0.2$ & 0.000190920959 & 0.002006552384 \\
$n=0.5$ & 0.021214895081 & 0.049202206137 \\
$n=1.0$ & 0.042000490258 & 0.086000793253 \\
$n=3.0$ & 0.049111111111 & 0.0984444444444 \\
$n \rightarrow \infty$ & 0.05 & 0.1 \\
\hline$A_{2}(m, n)$ & $m=0.6$ & $m=0.8$ \\
\hline$n=0.1$ & 0.000191411030 & 0.014197530933 \\
$n=0.2$ & 0.014197530723 & 0.069964723451 \\
$n=0.5$ & 0.091202696394 & 0.155215688334 \\
$n=1.0$ & 0.134000793253 & 0.188000490258 \\
$n=3.0$ & 0.148222222222 & 0.1986666666667 \\
$n \rightarrow \infty$ & 0.15 & 0.2 \\
\hline$A_{2}(m, n)$ & $m=1.0$ & \\
\hline$n=0.1$ & 0.25 & \\
$n=0.2$ & 0.25 & \\
$n=0.5$ & 0.25 & \\
$n=1.0$ & 0.25 & \\
$n=3.0$ & 0.25 & \\
$n \rightarrow \infty$ & 0.25 & \\
\hline & & \\
& &
\end{tabular}

Table 4. Some pertinent values of $A_{3}(m, n)$.

\begin{tabular}{ccc}
\hline$A_{3}(m, n)$ & $m=0.2$ & $m=0.4$ \\
\hline$n=0.1$ & 0.000000000024 & 0.000000069101 \\
$n=0.2$ & 0.000035327336 & 0.000433859886 \\
$n=0.5$ & 0.006103155817 & 0.014855370235 \\
$n=1.0$ & 0.014568142536 & 0.030170107421 \\
$n=10.0$ & 0.018761210176 & 0.037533695300 \\
$n \rightarrow \infty$ & 0.018806319452 & 0.037612638903 \\
\hline$A_{3}(m, n)$ & $m=0.6$ & $m=0.8$ \\
\hline$n=0.1$ & 0.000035396432 & 0.003643246632 \\
$n=0.2$ & 0.003643246609 & 0.021612750820 \\
$n=0.5$ & 0.029423512772 & 0.053967597532 \\
$n=1.0$ & 0.047864441715 & 0.068758538572 \\
$n=10.0$ & 0.056328732577 & 0.075157603731 \\
$n \rightarrow \infty$ & 0.056418958355 & 0.075225277806 \\
\hline$A_{3}(m, n)$ & $m=1.0$ & \\
\hline$n=0.1$ & 0.094031597258 & \\
$n=0.2$ & 0.094031597258 & \\
$n=0.5$ & 0.094031597258 & \\
$n=1.0$ & 0.094031597258 & \\
$n=10.0$ & 0.094031597258 & \\
$n \rightarrow \infty$ & 0.094031597258 & \\
\hline & &
\end{tabular}

Table 5. Some pertinent values of $A_{4}(m, n)$.

\begin{tabular}{cll}
\hline$A_{4}(m, n)$ & \multicolumn{1}{c}{$m=0.2$} & \multicolumn{1}{c}{$m=0.4$} \\
\hline$n=0.1$ & 0.000000000003 & 0.000000009433 \\
$n=0.2$ & 0.000006218729 & 0.000088122390 \\
$n=0.5$ & 0.001593566218 & 0.004058221022 \\
$n=1.0$ & 0.004479320915 & 0.009380313240 \\
$n=10.0$ & 0.006230022933 & 0.012465038033 \\
$n \rightarrow \infty$ & 0.00625 & 0.0125 \\
\hline$A_{4}(m, n)$ & \multicolumn{1}{c}{$m=0.6$} & $m=0.8$ \\
\hline$n=0.1$ & 0.000006228163 & 0.000863879708 \\
$n=0.2$ & 0.000863879706 & 0.006043996579 \\
$n=0.5$ & 0.008537541937 & 0.016738212792 \\
$n=1.0$ & 0.015144646573 & 0.022253987582 \\
$n=10.0$ & 0.018710039467 & 0.024970025400 \\
$n \rightarrow \infty$ & 0.01875 & 0.025 \\
\hline$A_{4}(m, n)$ & $m=1.0$ & \\
\hline$n=0.1$ & 0.03125 & \\
$n=0.2$ & 0.03125 & \\
$n=0.5$ & 0.03125 & \\
$n=1.0$ & 0.03125 & \\
$n=10.0$ & 0.03125 & \\
$n \rightarrow \infty$ & 0.03125 & \\
\hline & &
\end{tabular}


Table 6. Some pertinent values of $A_{5}(m, n)$.

\begin{tabular}{ccc}
\hline$A_{5}(m, n)$ & $m=0.2$ & $m=0.4$ \\
\hline$n=0.1$ & 0.0000000000003 & 0.000000001250 \\
$n=0.2$ & 0.000001047128 & 0.000016949350 \\
$n=0.5$ & 0.000384260310 & 0.001021389876 \\
$n=1.0$ & 0.001250563532 & 0.002647668975 \\
$n=10.0$ & 0.001873122349 & 0.003748120914 \\
$n \rightarrow \infty$ & 0.001880631945 & 0.003761263890 \\
\hline$A_{5}(m, n)$ & $m=0.6$ & $m=0.8$ \\
\hline$n=0.1$ & 0.000001048378 & 0.000191548722 \\
$n=0.2$ & 0.000191548721 & 0.001556875424 \\
$n=0.5$ & 0.002271953408 & 0.004732477401 \\
$n=1.0$ & 0.004348217091 & 0.006530073094 \\
$n=10.0$ & 0.005626873035 & 0.007511258312 \\
$n \rightarrow \infty$ & 0.005641895835 & 0.007522527781 \\
\hline$A_{5}(m, n)$ & $m=1.0$ & \\
\hline$n=0.1$ & 0.009403159726 & \\
$n=0.2$ & 0.009403159726 & \\
$n=0.5$ & 0.009403159726 & \\
$n=1.0$ & 0.009403159726 & \\
$n=10.0$ & 0.009403159726 & \\
$n \rightarrow \infty$ & 0.009403159726 & \\
\hline
\end{tabular}

Table 7. Values of $A_{r}(1, n)$ for $r=6$ - 19.

\begin{tabular}{ll}
$r=6$ & 0.002604166667 \\
$r=7$ & 0.000671654266 \\
$r=8$ & 0.000162760417 \\
$r=9$ & 0.000037314126 \\
$r=10$ & 0.000008138021 \\
$r=11$ & 0.000001696097 \\
$r=12$ & 0.0000000339084 \\
$r=13$ & 0.000000065234 \\
$r=14$ & 0.000000012110 \\
$r=15$ & 0.000000002174 \\
$r=16$ & 0.000000000378 \\
$r=17$ & 0.000000000064 \\
$r=18$ & 0.000000000011 \\
$r=19$ & 0.000000000002 \\
\hline
\end{tabular}

n, i.e.,

$$
A_{r}\left(m_{2}, n\right)>A_{r}\left(m_{1}, n\right) \text { for } m_{2}>m_{1} \geq 0 .
$$

- The functions have maximum values for $m=1$. Moreover, they have the same values for $m=1$ and any value of $n$. These values are as follows:

$$
A_{r}(1, n)=\mathrm{i}^{r} \operatorname{erfc} 0
$$

or

$$
A_{r}(1, n)=\left[2^{r} \Gamma(r / 2+1)\right]^{-1}
$$

where $\Gamma$ is the gamma function.

- When $n$ is larger, the function $A_{r}(m, n)$ is also larger for any fixed value of $m$, i.e.,

$$
A_{r}\left(m, n_{2}\right)>A_{r}\left(m, n_{1}\right) \text { for } n_{2}>n_{1} .
$$

For large values of $n$, it approximately varies linearly with $m$, i.e.,

$$
A_{r}(m, n) \cong\left(\mathrm{i}^{r} \operatorname{erfc} 0\right) m
$$

$A_{r}(m, n)$ is more linear than $A_{r+1}(m, n)$.

- For $n \rightarrow \infty$, it is a linear function of $m$, i.e.,

$$
A_{r}(m, \infty)=\left(i^{r} \operatorname{erfc} 0\right) m \text {. }
$$

\section{REFERENCES}

[1] H. V. Ersoy, "Unsteady Flow Due to a Sudden Pull of Eccentric Rotating Disks," International Journal of Engineering Science, Vol. 39, No. 3, 2001, pp. 343-354. doi:10.1016/S0020-7225(00)00040-9

[2] M. Abramowitz and I. A. Stegun, "Handbook of Mathematical Functions,” Dover Publications, New York, 1972. 